TRANSACTIONS OF THE

AMERICAN MATHEMATICAL SOCIETY

Volume 275, Number 1, January 1983

\title{
ON THE TRIANGULATION OF STRATIFIED SETS AND SINGULAR VARIETIES
}

BY

F. E. A. JOHNSON ${ }^{1}$

\begin{abstract}
ABSTRACr. We show that every compact stratified set in the sense of Thom can be triangulated as a simplicial complex. The proof uses that author's description of a stratified set as the geometric realisation of a certain type of diagram of smooth fibre bundles and smooth imbeddings, and the triangulability of smooth fibre bundles.

As a consequence, we obtain proofs of the classical triangulation theorems for analytic and subanalytic sets, and a correct proof of Yang's theorem that the orbit space of a smooth compact transformation group is triangulable.
\end{abstract}

0. Introduction. For many years, the category of smooth manifolds and smooth maps was deficient in that it lacked the formal notion of "singular" objects, even though such objects occur naturally when considering singularities of smooth mappings. This defect was repaired by Thom in the introduction in [15] of stratified sets, which can be thought of as having the same relation to smooth manifolds as singular algebraic varieties have to nonsingular ones.

In this paper, we present the extension to compact stratified sets of Whitehead's $C^{1}$ triangulation theorem [17].

Some fifty years or so ago, there began to appear a sequence of papers concerned with the problem of triangulating various types of singular varieties. Algebraic sets were triangulated by Van der Waerden [16], analytic sets by Koopman and Brown, and independently by Lefschetz and Whitehead [11, 12]. To the modern eye, these old proofs look messy and ad hoc. Certainly they have never enjoyed the same secure status within Topology as Whitehead's $C^{1}$ triangulation theorem of 1940. Examination of these old proofs leaves the impression that all the really difficult work involved in triangulation goes into presenting the candidates for triangulation in some reasonable preliminary form. Such a decomposition into regular pieces was accomplished with great rigour by Whitney in [18]. Following Whitney's work, Thom introduced the formal notion of a stratified set. We were led to suspect that triangulability is a fairly easy consequence of being stratified, and in this we have been justified. We shall prove that every compact stratified set admits a triangulation. In consequence, we recover the compact cases of all the classical results mentioned above, and, moreover, bring them in uniform fashion under the dominion of the $C^{1}$ triangulation theorem. We also recover the compact cases of the theorems

Received by the editors March 6, 1979 and, in revised form, September 14, 1981 and January 11, 1982. 1980 Mathematics Subject Classification. Primary 32C42, 54E60, 57R05. Secondary 55R65.

${ }^{1}$ Research supported by a grant from the Science Research Council and by National Science Foundation grant GP-364181X1. 
of Lojasiewicz [13], and Hironaka [5], the modern heirs to the old tradition. Moreover, "since it is well known, using the Slice Theorem, that if $G$ is a compact Lie group acting smoothly on the compact manifold $M$ then $M / G$ is a stratified set", we recover the triangulation theorem of Yang [19]. This is important, since it is now clear that Yang's original proof is incomplete, relying upon a false triangulation criterion of Cairns.

The theorem proved in this paper was first proved in the author's thesis [7], written under the guidance of Professor C. T. C. Wall. The delay in publishing these results was occasioned by my attempts to reduce the length of the companion paper [10], on which the present paper relies, from the monstrous proportions it attained in earlier drafts to its present mere 56 pages of typescript.

The triangulation theorem proved here is surely true under the more general hypothesis that the stratified set be a countable, locally finite union of manifolds subject to a suitable attaching condition. The necessary preliminary seems to be a corresponding extension of the main result of [10].

Since the author's thesis of 1972, a number of other papers proving similar results have appeared. At the editor's request I will review them briefly. In apparent historical order, the versions of which I am aware are:

[A] E. Hendricks, Triangulation of stratified sets, Thesis, MIT, 1973.

[B] M. Kato, Elementary topology of analytic sets, Sûgaku 25 (1973), 38-51. Japanese

[C] W. Ullman, Triangulability of abstract prestratified sets and stratification of the orbit space of a G-manifold, Thesis, Bonn, 1973.

[D] T. Matumoto, Equivariant stratification of a differentiable transformation group, Preprint, 1977.

[E] M. Goresky, Triangulation of stratified objects, Proc. Amer. Math. Soc. 72 (1978), 193-200.

[F] A. Verona, Triangulation of stratified fibre bundles, Manuscripta Math. 30 (1980), 425-445.

No doubt the above ordering does somebody some injustice, but this is not intentional. Of these, [A, $\mathbf{C}$ and $\mathbf{D}$ ] are apparently unpublished. In addition to these I might also mention the rather less general papers of Illman [6] and Hardt (Triangulation of subanalytic sets and proper light subanalytic maps, Invent. Math. 38 (1977), 207-217). I should also mention my own preprint with the same title as the present paper which I wrote at the Institute for Advanced Study in 1973/74 and which has circulated underground since then. This preprint is broadly very similar to the present paper, differing only by being less detailed.

Of the papers $[\mathbf{A}-\mathbf{F}]$ above, I have had the opportunity of reading only $[\mathbf{D}, \mathbf{E}$ and F]. With exception of [D], all the papers deal with triangulation of stratified sets in the sense of Mather. For reasons of purity, and out of a general preference for abstraction, I have always used Thom's original definition. It seems clear, at least in the compact case, that every abstract stratified set in the sense of Thom can be imbedded in some Euclidean space as a Whitney prestratification in the sense of Mather. Perhaps one day somebody will trouble to write down a proof. As far as can 
be judged from reports, with the exception of [F and $\mathbf{D}$ ], all the triangulation proofs are basically of the "piecing together of local triangulations" type, and so to some extent still in the line of development from Van der Waerden's paper [16].

Verona's paper $[\mathbf{F}]$ is somewhat more global than the others and, though considerably less formalised than the present paper, has a number of points of similarity with our account.

Matumoto's paper does deal with Thom stratifications, but it can really be seen only as a sketch proof. It is very similar to an early attempted proof of mine (circa August 1970). Matumoto dismisses all the difficulties surrounding simultaneous triangulation of manifolds and maps in a single sentence (p. 5 of [D]). In the end, I found it necessary to write [9].

Finally, it is a pleasure to be able to thank a number of people and institutions. Thanks to Terry Wall, who listened to my first ideas on the subject and put me straight at a number of crucial points. Thanks to Tony Solomonides, who helped me run a seminar on these ideas at King's College London in 1977, and to Professor Armand Borel and Gopal Prasad who suggested the application to $S$-arithmetic groups given in §2. Thanks especially to the Institute for Advanced Study, Princeton, and to the Science Research Council and the National Science Foundation for financial support whilst I wrote and revised portions of this work. Lastly, thanks to the referee, whose diligent attention to detail brought to light a number of indexing errors.

1. Triangulation of generalised fibre bundles. We explain briefly our conventions concerning smooth manifolds and diagrams of smooth manifolds and smooth maps.

It is essential that we allow our manifolds to have corners. In setting up our definition, we generally follow Cerf [3], with the difference that our local models are allowed to be just slightly more complicated: whereas Cerf's local models are the spaces $\mathbf{R}^{n} \times \mathbf{R}_{+}^{m}$, we allow extra factors of the form $\overline{\mathbf{R}^{n}-\left(\mathbf{R}_{+}\right)^{n}}$. Precisely, by a local model we shall mean a product of the form $C_{1} \times \cdots \times C_{k}$, where each $C_{i}$ is one of $\mathbf{R}, \mathbf{R}_{+}$or $\mathbf{R}^{p}-\left(\mathbf{R}_{+}\right)^{p}$. As might be expected, a smooth manifold is then a pair $(X, \Lambda)$ where $X$ is a paracompact Hausdorff space, and $\Lambda$ is a maximal collection of charts of the form $h: U \rightarrow V$ with $U$ open in $X, V$ an open subset of a local model, such that the domains of charts in $\Lambda$ cover $X$, and such that any two charts are $C^{\infty}$ compatible in the usual sense, namely that if $h_{i}: U_{i} \rightarrow V_{i}$ are charts in $\Lambda, i=1,2$, then $h_{2} \circ h_{1}^{-1}: h_{1}\left(U_{1} \cap U_{2}\right) \rightarrow h_{2}\left(U_{1} \cap U_{2}\right)$ is a $C^{\infty}$ diffeomorphism. In particular, we take "smooth" to mean " $C^{\infty}$ smooth". If $M$ is a smooth manifold, then any point $x$ of $M$ has a neighbourhood diffeomorphic to a local model by a diffeomorphism taking $x$ to 0 .

We denote by DIFF the category of smooth manifolds and $C^{\infty}$ smooth maps.

Much of the time we shall work in functor categories. We adopt the convention that if $\mathcal{C}$ and $\mathscr{D}$ are categories, [C, $\mathscr{D}]$ denotes the category whose objects are functors $C \rightarrow \mathscr{D}$, and whose morphisms are natural transformations.

In practice, we shall resort to a very limited range of indexing categories. For a positive integer $n, 2^{n}$ will denote the set of subsets of $\{0, \ldots, n-1\}$, partially ordered by taking $x \leqslant y$ if $y \subset x$. As a category, there is at most one morphism 
between any two objects of $2^{n}$, with $\operatorname{Hom}(x, y) \neq \varnothing$ iff $x \supset y . \mathbf{K}_{n}$ will denote the full subcategory of $2^{n+1}$ obtained by omitting the empty set. We take $2^{0}$ to be the trivial category with one object. The nerves of $2^{n}, \mathbf{K}_{n}$ are, respectively, the $n$-cube and the barycentric subdivision of the $n$-simplex. $\mathbf{K}_{n+1}$ contains two canonical copies of $\mathbf{K}_{n}, \mathbf{K}_{n}^{+}$and $\mathbf{K}_{n}^{-}$, and a copy, $C^{n+1}$, of $2^{n+1} . \mathbf{K}_{n}^{-} \subset \mathbf{K}_{n+1}$ is induced from the inclusion $\{0, \ldots, n\} \subset\{0, \ldots, n+1\} . C^{n+1}$ is the image of the functor $2^{n+1} \rightarrow \mathbf{K}_{n+1}$, $A \mapsto A \cup\{n+1\}$, and $\mathbf{K}_{n}^{+}$is the image of $\mathbf{K}_{n}$ under this functor. A morphism $\varphi$ : $\mathbf{K}_{n}^{+} \rightarrow \mathbf{K}_{n}^{-}$identifies the copies thus; $\varphi(A)=A \backslash\{n+1\}$.

Definition. By a smooth manifold $n$-ad we mean a functor $M: 2^{n} \rightarrow$ DIFF such that each $M(x)$ is nonempty and

(i) if $x \supset y$ and $\operatorname{card}(x)=\operatorname{card}(y)+1$, then $M(x)$ is a closed smooth submanifold of codimension 1 in $M(y)$, with $M(x)$ actually contained in the boundary of $M(y)$, and such that the structural map $M(x) \rightarrow M(y)$ is an imbedding, and

(ii) for all $x, y, M(x \cup y)=M(x) \cap M(y)$, the intersection being transverse.

The restriction of an $n$-ad to $\mathbf{K}_{n-1}$ is called its formal boundary. In general, by a formal boundary of type $(n-1)$, we mean a functor $\delta M: \mathbf{K}_{n-1} \rightarrow$ DIFF which is the restriction to $\mathbf{K}_{n-1}$ of some smooth manifold $n$-ad. In particular, each of the structure maps of a formal boundary must be a smooth imbedding.

The boundary of a smooth manifold with corners is not, in general, smooth, though it can be smoothed [4]. We wish to consider what happens to a formal boundary after the ambient $n$-ad has been smoothed. We do this by looking at the operation of introducing corners.

Definition. Let $V$ be a smooth manifold, possibly with corners. By a corner structure of type $(n-1)$ on $V$ we mean a pair $(M, h)$, where $M: 2^{n} \rightarrow$ DIFF is a smooth manifold $n$-ad, and $h$ is a homeomorphism between the union of proper faces of $M$ and $V$ thus;

$$
h: \bigcup_{\alpha \neq \varnothing} M(\alpha) \rightarrow V
$$

and such that, for each $\alpha \in \mathbf{K}_{n-1}$, i.e. each $\alpha \neq \varnothing, h: M(\alpha) \rightarrow V$ is a smooth imbedding. Thus a corner structure on $V$ essentially imposes the structure of a formal boundary on $V$.

Given a corner structure $(M, h)$ on a manifold $V$, the functor $\mathbf{K}_{n-1} \rightarrow$ DIFF given by $\alpha \mapsto h(M(\alpha))$, is called the associated flattened corner structure on $V$. It is called flattened since $V$ has fewer corners than $M$. It is important to notice that a flattened corner structure is not itself a formal boundary. It is what a formal boundary becomes after its corners have been smoothed.

By a generalised smooth fibre bundle of type $n$, we mean a morphism $f: X \rightarrow Y$ in $\left[\mathbf{K}_{n}\right.$, DIFF] such that, for each $v \in \mathbf{K}_{n}, f(V): X(v) \rightarrow Y(v)$ is a smooth, locally trivial fibre bundle.

If $X: \mathcal{C} \rightarrow$ DIFF is a functor, then by a P.D. triangulation of $X$ we mean a pair $(L, h)$ where $L: \circlearrowright \rightarrow \mathrm{PL}$ is a functor and $h: L \rightarrow X$ is a natural transformation such that, for each $v \in \mathcal{C}, h(v): L(V) \rightarrow X(v)$ is a piecewise differentiable (= P.D.) triangulation. As is well known, each smooth manifold $n$-ad admits a P.D. triangulation. This can be obtained by induction on $n$ using Whitehead's original theorems, 
and starting from the case $n=0$, which is just the assertion that a smooth manifold admits a P.D. triangulation. We refer the reader to the argument in $\$ 3$ of [9]. Also every formal boundary admits a P.D. triangulation. Moreover, since at the piecewise differentiable level formal boundaries are indistinguishable from flattened corner structures, we see that every flattened corner structure admits a P.D. triangulation.

Next we introduce the diagram theoretic analogue of a stratified set. Such diagrams we call "normal". The definition is inductive.

Definition. By a normal diagram of type 0 , we mean a smooth manifold, or, equivalently, a functor $X: \mathbf{K}_{0} \rightarrow$ DIFF. By a normal diagram of type $n$, we mean a functor $X: \mathbf{K}_{n} \rightarrow$ DIFF such that:

(a) the restriction of $X$ to $\mathbf{K}_{n-1}^{-}$is normal of type $(n-1)$,

(b) the restriction of $X$ to $\mathbf{K}_{n-1}^{+}$is either a flattened corner structure on $X(n)$ itself or else is a flattened corner structure on some smooth submanifold $V$ of codimension 1 in $X(n)$ such that $V$ is actually contained in the boundary of $X(n)$ thus $V \subset \partial X(n)$

(c) each structure morphism $X(\alpha) \rightarrow X(n), n \in \alpha$, is an inclusion as a smooth submanifold,

(d) $X(\varphi): X_{\mid \mathbf{K}_{n-1}^{+}} \rightarrow X_{\mid \mathbf{K}_{n-1}^{-}}$is a generalised smooth fibre bundle such that, if $A \subset B, A, B \in \mathbf{K}_{n-1}^{-}$, then $X(\varphi)_{(A)}^{-1}(X(B))=X(B \cup\{n\})$.

A normal diagram will be called compact iff each vertex space is compact.

The obscurity of condition (b) in the above definition has been remarked upon to me by the referee. To clarify this point, note that we are trying to define a functor $X$ : $\mathbf{K}_{n} \rightarrow$ DIFF. To do this is equivalent to specifying (i) $X_{\mid \mathbf{K}_{n-1}^{-}}$, (ii) $X_{\mid \mathbf{K}_{n-1}^{+}}$, (iii) $X(\varphi)$ : $X_{\mid \mathbf{K}_{n-1}^{+}} \rightarrow X_{\mid \mathbf{K}_{n-1}^{-}}$, (iv) the space $X(n)$ and (v) the morphisms $X(\alpha) \rightarrow X(n)$ where $n \in \alpha$. The points at issue are (ii), (iv) and (v). (iv) is easy to clear up; $X(n)$ must be a smooth manifold. Beyond this there are two possibilities: either the union $\cup_{n \in \alpha ; \alpha \neq\{n\}} X(\alpha)$ constitutes the whole of $X(n)$, the morphisms $X(\alpha) \rightarrow X(n), n \in \alpha$, $\{n\} \neq \alpha$, are just inclusions, and $X_{\mid \mathbf{K}_{n-1}^{+}}$is a flattened corner structure on $X(n)$ or the union $\bigcup_{\{n\} \nsubseteq \alpha} X(\alpha)$ is only a smooth submanifold $V$ of $X(n)$, and $V$ is contained in $\partial X(n)$, with codimension 0 in $\partial X(n)$, codimension 1 in $X(n)$ itself, and $X_{\mid \mathbf{K}_{n-1}^{+}}$is a flattened corner structure on $V$.

The apparent disparity of the two cases disappears at the level of geometric realisations. That is, if the geometric realisation of $X$ is taken, the highest dimension occurring is the same in each case, namely, $\operatorname{dim}(X\{0, \ldots, n\})+n$, or equivalently, $\operatorname{dim}(X\{n-1, n\})+1$. The reason for having both possibilities is a technical one arising from the companion paper [10]. It would be possible, at the cost of greater complication in [10], to eliminate the first of the above possibilities altogether. The vertex space $X(n)$ must then be replaced by $X(n) \times[0,1]$, and the morphisms $X(\alpha) \rightarrow X(n)$ by the obvious imbeddings into $X(n) \times\{0\}$. Note that, up to homeomorphism, the geometric realisation is not affected.

To proceed, we introduce a filtration into the categories $\mathbf{K}_{n}$; let $\mathscr{Q}_{s, t}$ be the following full subcategory of $\mathbf{K}_{s+t} \cdot \mathscr{D}_{0, n}=\mathbf{K}_{n}$, and for $1 \leqslant k, \mathscr{D}_{k, n}=\left\{A \in \mathbf{K}_{n+k}\right.$ : $A \nsubseteq\{n+1, \ldots, n+k\}\}$. We have inclusions as follows:

$$
\mathscr{D}_{k+n, 0} \subset \mathscr{D}_{k+n-1,1} \subset \cdots \subset \mathscr{D}_{k+1, n-1} \subset \mathscr{D}_{k, n} \subset \cdots \subset \mathscr{D}_{0, k+n} .
$$


Moreover, in each case, we have a retraction $r_{k, n}: \mathscr{D}_{k, n} \rightarrow \mathbf{K}_{n}$ defined by

$$
r_{k, n}(A)=A \cap\{0, \ldots, n\} \text {. }
$$

Note that each $r_{k, n}$ is a functor.

For example, the filtration on $\mathbf{K}_{2}$ is as follows, with retractions indicated by arrows.
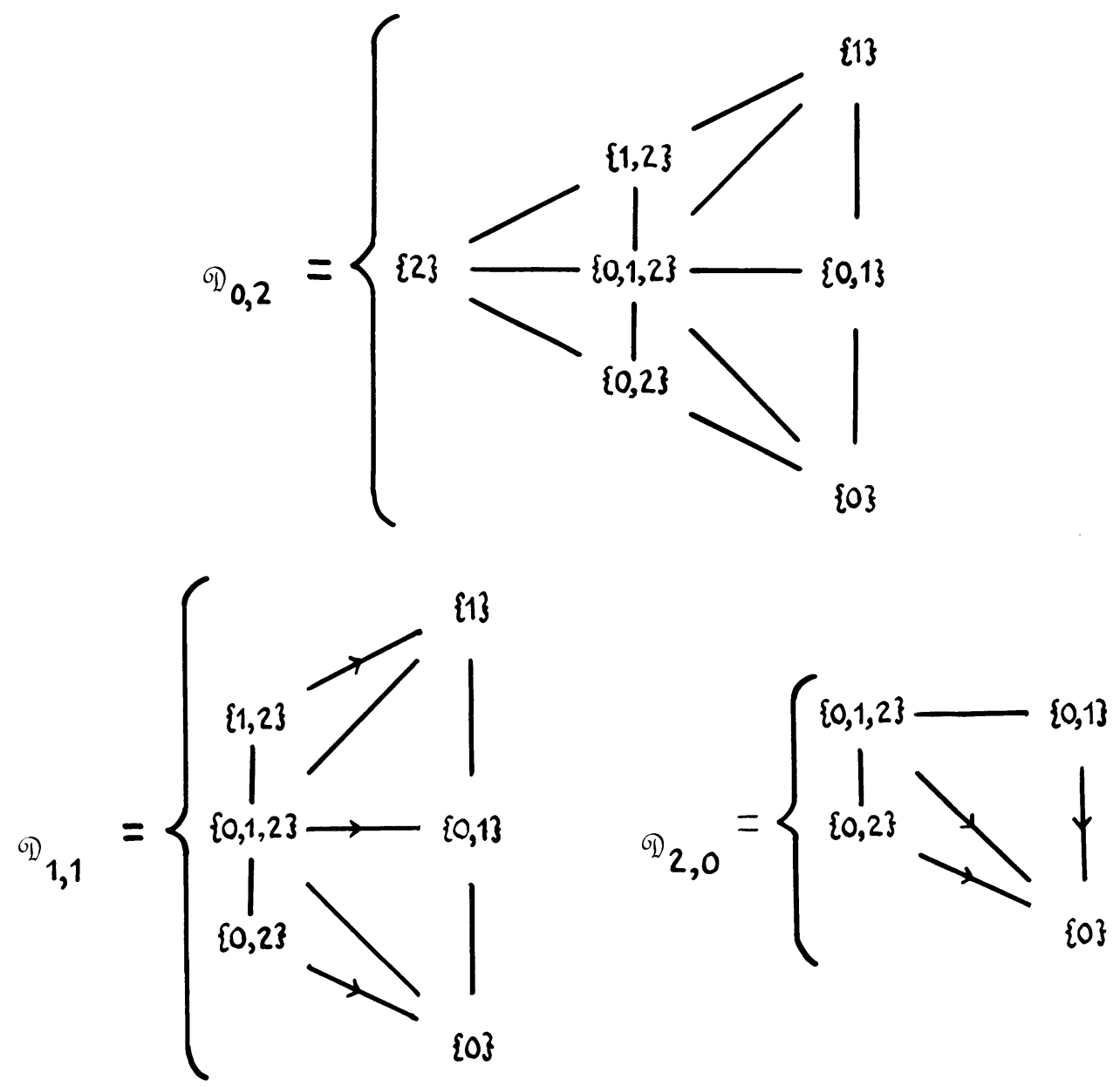

The reader may find it helpful to work out the filtration on $\mathbf{K}_{3}$.

The author finds it psychologically useful to observe that the retraction $r_{k, n}$ : $\mathscr{D}_{k, n} \rightarrow \mathbf{K}_{n}$ is equivalent to projection from a product. In fact, defining $s_{k, n}$ : $\mathscr{D}_{k, n} \rightarrow 2^{k}$ by $s_{k, n}(A)=\{a-n-1: a \in A \cap\{n+1, \ldots, n+k\}\}$, it is easy to see that $\xi_{k, n}: \mathscr{D}_{k, n} \rightarrow \mathbf{K}_{n} \times 2^{k}$ defined by $\xi_{k, n}(A)=\left(r_{k, n}(A), s_{k, n}(A)\right)$ is an isomorphism of categories and makes the diagram below commute:

$$
\begin{array}{cccc}
\mathscr{D}_{k, n} & \stackrel{\xi_{k, n}}{\rightarrow} & & \mathbf{K}_{n} \times 2^{k} \\
& \searrow r_{k, n} & & \swarrow \text { project } \\
& & \mathbf{K}_{n}
\end{array}
$$


The product structure $\mathscr{D}_{1,1}=\mathbf{K}_{1} \times 2^{1}$ is apparent in the filtration of $\mathbf{K}_{2}$ illustrated above. However, we shall make no explicit use of this product structure in what follows.

Definition. A generalised smooth $k$-ad bundle of type $n$ (or $(k, n)$-bundle) is a triple $(X, Y, \Lambda)$ where

(i) $X$ is a smooth manifold $(k+n+1)-a d$,

(ii) $Y$ is a normal diagram of type $n$ and

(iii) $\Lambda: X_{\mid \Phi_{k, n}} \rightarrow Y$ is a morphism of smooth diagrams defined over the retraction $r_{k, n}: \mathscr{D}_{k, n} \rightarrow \mathbf{K}_{n}$, such that $\Lambda_{(A)}: X(A) \rightarrow Y\left(r_{k, n}(A)\right)$ is a smooth fibre bundle, for all $A \in \mathscr{D}_{k, n}$, and such that, if $A \subset B, A \neq \varnothing, B \subset\{0, \ldots, n\}$, then $\Lambda_{(A)}^{-1}(B)=X(B)$.

The formal boundary of such a $(k, n)$-bundle is defined to be $\left(\delta_{k} X, Y, \delta_{k} \Lambda\right)$, where $\delta_{k} X$ is the restriction of $X$ to the category

$$
\partial \mathscr{D}_{k, n}=\left\{A \in \mathscr{D}_{k, n}: A \cap\{n+1, \ldots, n+k\} \neq \varnothing\right\},
$$

taking $\partial \mathscr{Q}_{k, 0}$ to be empty, by convention, and where $\delta_{k} \Lambda$ is the restriction of $\Lambda$ to $\delta_{k} X$.

We now explain how to associate with each $(k, n+1)$-bundle a canonical $(k+1, n)$-bundle. Start with a diagram $Z$ over $\mathbf{K}_{n+1}$. Then $Z$ gives rise to a "retraction" $r(Z): Z_{\mid \mathscr{D}_{1, n}} \rightarrow Z_{\mid \mathbf{K}_{n}}$ defined over the retraction $r_{1, n}: \mathscr{D}_{1, n} \rightarrow \mathbf{K}_{n}$ as follows. The maps $r(Z)(A)$ for $A \in \mathscr{D}_{1, n}$ are defined to be the structure morphisms of $Z, r(Z)(A): Z(A) \rightarrow Z(A \cap\{0, \ldots, n\})$, induced by $A \supset A \cap\{0, \ldots, n\}$. In the special case where $A \subset\{0, \ldots, n\}$, then $r(Z)(A)$ is just the identity on $Z(A)$. If $Z$ is a normal diagram and $A \nsubseteq\{0, \ldots, n\}$, then

$$
r(Z)(A): Z(A) \rightarrow Z(A \cap\{0, \ldots, n\})=Z(A \backslash\{n+1\})
$$

is a smooth fibre bundle.

Definition. Given a $(k, n+1)$-bundle $(X, Y, \Lambda)$, we define the associated $(k+1, n)$-bundle to be the triple $\left(X, Y_{\mid \mathbf{K}_{n}^{-}}, R(\Lambda)\right)$ where $R(\Lambda)$ is the composition $R(\Lambda)=r(Y) \circ \Lambda_{k+1, n}$,

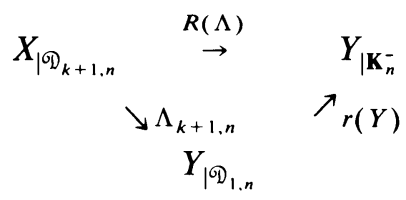

where $\Lambda_{k+1, n}$ is the restriction of $\Lambda$ to $\mathscr{D}_{k+1, n}$. In explicit terms, the $R(\Lambda)_{(A)}$ are given by

$$
R(\Lambda)_{(A)}=\left\{\begin{aligned}
\Lambda_{(A)} & \text { if } A \subset\{0, \ldots, n\} \\
r(Y)\left(r_{k, n+1}(A)\right) \circ \Lambda_{(A)} & \text { if } A \nsubseteq\{0, \ldots, n\} \text { or, } \\
& \text { equivalently, }
\end{aligned}\right.
$$

It is straightforward to check that $\left(X, Y_{\mid \mathbf{K}_{n}^{-}}, R(\Lambda)\right)$ really is a $(k+1, n)$-bundle. First of all, note that $\mathscr{D}_{k+1, n}=r_{k, n+1}^{-1}\left(\mathscr{D}_{1, n}\right)$ and that $r_{k+1, n}=r_{1, n} \circ\left(r_{k, n+1 \mid \mathscr{D}_{k+1, n}}\right)$. Hence $\Lambda_{k+1, n}: X_{\mid \mathscr{D}_{k+1, n}} \rightarrow Y_{\mid \mathscr{D}_{1, n}}$ is well defined, and moreover, the composite $R(\Lambda)$ $=r(Y) \circ \Lambda_{k+1, n}$ is defined over the retraction $r_{k+1, n}=r_{1, n} \circ\left(r_{k, n+1 \mid \mathscr{D}_{k+1, n}}\right)$. Since $Y$ 
is a normal diagram, it follows that $Y_{\mid \mathbf{K}_{n}^{-}}$is also a normal diagram. Finally, each $R(\Lambda)_{(A)}$ is a composition of smooth fibre bundles, hence is a smooth fibre bundle, so that $\left(X, Y_{\mid \mathbf{K}_{n}^{-}}, R(\Lambda)\right)$ is a $(k+1, n)$-bundle.

TheOREM 1.1. Let $(X, Y, \Lambda)$ be a $(k, n)$-bundle with $X, Y$ compact. Any P.D. triangulation of $\delta_{k} \Lambda: \delta_{k} X \rightarrow Y$ extends to one of $\Lambda: X_{\mid \mathscr{D}_{k, n}} \rightarrow Y$.

Proof. Let $P(k, n)$ be the above statement. It suffices to show that $P(k, 0)$ is true for all $k$, and that $P(k+1, n-1)$ implies $P(k, n)$ for $n \geqslant 1$.

$P(k, 0)$ is true. Let $Y$ be a smooth compact manifold, $X$ a smooth compact manifold $(k+1)$-ad, and let $\Lambda: X(0) \rightarrow Y$ be a smooth locally trivial fibre bundle whose restrictions $\Lambda: X(A) \rightarrow Y$ for $0 \in A$ are also locally trivial. Then $(X, Y, \Lambda)$ is exactly a $(k, 0)$-bundle. Recall that $\mathscr{D}_{k, 0}=\left\{A \in \mathbf{K}_{k}: 0 \in A\right\}$ and that $\partial \mathscr{D}_{k, 0}=\{A$ $\left.\in \mathscr{Q}_{k, 0}:\{0\} \neq A\right\}$. Notice that the collection $(X(A))_{0 \in A}$ is really a smooth manifold $k$-ad in disguised form. To put it in canonical form it is necessary to re-index as follows:

$$
\hat{X}(\varnothing)=X(0) \text { and } \hat{X}((A \cap\{1, \ldots, k\})-1)=X(A) \text { for } 0 \neq A,
$$

when $\hat{X}$ becomes a smooth manifold $k$-ad in canonical form. However, in what follows, we shall leave $X$ in its original disguised form.

Assume, as hypothesis $P(k, 0)$, that we are given P.D. triangulations $h_{1}: L \rightarrow Y$, $\delta h_{2}: \delta K \rightarrow \delta_{k} X$ and a PL map $\delta p: \delta k \rightarrow L$ such that $\delta h=\left(\delta h_{2}, h_{1}\right)$ is a P.D. triangulation $\delta h:(\delta K, L, \delta p) \rightarrow\left(\delta_{k} X, Y, \delta_{k} \Lambda\right)$. We must show that we can extend $\delta K$ to a polyhedron $K$ and $\delta h_{2}$ to a P.D. triangulation $h_{2}: K \rightarrow X(0)$ such that $p=h_{1}^{-1} \Lambda h_{2}: K \rightarrow L$ is a PL map. But this is precisely the conclusion of Theorem (4.6) of [9] (Relative Triangulability of smooth fibre bundles). Hence $P(k, 0)$ is true for all $k$.

$P(k+1, n-1) \Rightarrow P(k, n)$. Let $(X, Y, \Lambda)$ be a $(k, n)$-bundle. The domain of definition of $\Lambda$ is $X_{\mid \mathscr{D}_{k, n}}$. Set-theoretically, this is just $\cup_{0 \leqslant r \leqslant n} X(r)$, for if $A \in \mathscr{D}_{k, n}$, then $X(A) \subset X(r)$ for any $r \in A \cap\{0, \ldots, n\}$, whilst if $A \cap\{0, \ldots, n\}=\varnothing$, then $A \notin \mathscr{D}_{k, n}$. Now suppose, as the hypothesis of $P(k, n)$, that we are given a P.D. triangulation of the formal boundary $\delta_{k} \Lambda: \delta_{k} X \rightarrow Y$. In particular, we are given compatible triangulations of the family $X(\{n, n+r\}) \stackrel{\Lambda}{\rightarrow} Y(n), 1 \leqslant r \leqslant k$. The collection $(X(\{n\} \cup B))_{B \subset\{n+1, \ldots, n+k\}}$ is just a smooth manifold $k$-ad in disguised form. By Theorem (4.6) of [9], we may triangulate $\Lambda: X(n) \rightarrow Y(n)$ compatibly with the family $X(\{n, n+r\}) \stackrel{\Lambda}{\rightarrow} Y(n), 1 \leqslant r \leqslant k$, and hence compatibly with any

$$
X(\{n\} \cup B) \stackrel{\Lambda}{\rightarrow} Y(n), \quad B \subset\{n+1, \ldots, n+k\} .
$$

For each $A \subset\{0, \ldots, n\}$, with $n \in A, X(A)$ is now triangulated as a subcomplex of $X(n)$, since $X(A)=\Lambda_{(n)}^{-1}(Y(A))$, and $Y(A)$ is already triangulated as a subcomplex of $Y(n)$. Thus the original triangulations and the one constructed for $\Lambda: X(n) \rightarrow Y(n)$ now yield a triangulation of the formal boundary $\delta_{k+1} R(\Lambda)$ of the associated $(k+1, n-1)$-bundle $R(\Lambda)$. By hypothesis $P(k+1, n-1)$ we may complete the triangulation of $R(\Lambda)$ relative to that of $\delta_{k+1} R(\Lambda)$. In particular, we triangulate $R(\Lambda)_{(B)}: X(B) \rightarrow Y\left(r_{k+1, n-1}(B)\right)$ for $n \notin B$ compatibly with all previous 
triangulations. However, for $n \notin B, R(\Lambda)_{(B)}=\Lambda_{(B)}: X(B) \rightarrow Y\left(r_{k, n}(B)\right)$. Thus we have completed the triangulation of $\Lambda$ relative to the given triangulation of $\delta_{k} \Lambda$. Hence $P(k+1, n-1) \Rightarrow P(k, n)$. Q.E.D.

COROLlary 1.2. Let $\Lambda: X \rightarrow Y$ be a generalised smooth fibre bundle 0-ad of type $n$ with $Y$ compact normal, and $X$ a compact formal boundary. Then any P.D. triangulation of $Y$ can be lifted to one of $\Lambda$.

Proof. This is $P(0, n)$ above. Q.E.D.

CoROllaRY 1.3. Let $Y$ be a compact normal diagram over $K_{n}$. Then $Y$ admits a P.D. triangulation.

Proof. This follows easily from (1.2) by induction on $n$. One must also use the fact that P.D. triangulations of the formal boundary of an $n$-ad are extendible to the whole $n$-ad [9]. Q.E.D.

Actually, we can show, in the manner of the $C^{1}$ triangulation theorem for manifolds, that any two P.D. triangulations of a compact normal diagram are P.D. isotopic. The details are now rather routine, and we leave them to the reader to fill in.

2. Triangulation of compact stratified sets. A stratified set of type 0 is a smooth manifold. To construct a stratified set $X^{(k+1)}$ of type $k+1$, take a stratified set $X^{(k)}$ of type $k$, a smooth manifold $K$, a smooth submanifold $L$ of codimension 0 in $K$, and an "attaching map" $\alpha: L \rightarrow X^{(k)}$, and put $X^{(k+1)}=X^{(k)} \cup_{\alpha} K$. For the exact definition of "attaching" we refer the reader to [10 or 15]. Suffice it to say that $\alpha$ is not allowed to be an arbitrary continuous map, but must be, amongst other things, proper, continuous, and as close as possible to a smooth fibre bundle. $X^{(k+1)} \backslash X^{(k)}$ is called the stratum of height $k+1$. In general, we write $X^{(r)}$ for the union of strata of height $\leqslant r$.

THEOREM 2.1. A compact stratified set $X$ admits a triangulation by a finite simplicial complex so that $X^{(r)}$ is triangulated as a subcomplex, for each $r$.

Proof. If $C$ is an ordered simplicial complex, considered as a category, and if $F: \mathcal{C} \rightarrow$ TOP is a functor we denote by $\langle F\rangle$ the geometrical realisation of $F[8]$.

By the Main Theorem of [10], there is a normal diagram $\Psi(X)$ and a homeomorphism $h:\langle\Psi(X)\rangle \rightarrow X$ such that, for each $r$, there is a subdiagram $D^{(r)}$ of $\Psi(X)$ such that $h\left(\left\langle D^{(r)}\right\rangle\right)=X^{(r)} . \Psi(X)$ is necessarily compact since $X$ is. By (1.3) above, we can find a functor $F: \mathbf{K}_{n} \rightarrow$ PL, where $n$ is the type of $X$ and $\Psi(X)$, and a P.D. triangulation $k: F \rightarrow \Psi(X)$. Let $F^{(r)}$ be the subdiagram of $F$ corresponding to $D^{(r)}$. We get a homeomorphism of pairs $h \circ k_{*}:\left(\langle F\rangle,\left\langle F^{(r)}\right\rangle\right) \rightarrow\left(X, X^{(r)}\right)$, where $k_{*}$ : $\langle F\rangle \rightarrow\langle\Psi(X)\rangle$ is the induced homeomorphism of geometric realisations. To complete the proof, it suffices to triangulate $\langle F\rangle$ so that each $\left\langle F^{(r)}\right\rangle$ is triangulated as a subcomplex. That we can do precisely this is proved as the main theorem of [8]. Q.E.D. 
Recall that the $W$-objects of [15] are stratified sets in the sense of $[10,15]$. Hence we obtain

COROLlARY 2.2. Every compact $W$-object admits a triangulation.

Semialgebraic, semianalytic, and, more generally, subanalytic sets satisfy Whitney's regularity criteria [14]. Thus Corollary 2.2 above includes the compact cases of the "classical" triangulation theorems of Van der Waerden [16], Koopman-Brown and Lefschetz-Whitehead [11, 12], Lojasiewicz [13], and Hironaka [5], except they are now rehabilitated within the scope of Whitehead's $C^{1}$ triangulation theorem [17].

Our results can also be used to round off a theorem of Borel and Serre [2]. This application was suggested to me by Gopal Prasad and Professor Borel. Recall that in [2], Borel and Serre associate with each $S$-arithmetic group $\Gamma$ a compact space $X / \Gamma$, which is a $K(\Gamma, 1)$ when $\Gamma$ is torsion free. $X$ is the product of a Bruhat-Tits building with the Borel-Serre space $X_{\infty}$ obtained by adding corners to a classical symmetric space [1].

THEOREM 2.3. If $\Gamma$ is a torsion free $S$-arithmetic group, the Borel-Serre construction $X / \Gamma$ is triangulable as a finite simplicial complex.

Proof. It suffices to show that $X / \Gamma$ is a compact stratified set. Using our present notation, Borel and Serre show that $X / \Gamma$ is homeomorphic to the geometric realisation $\langle F\rangle$ of a certain functor $F: \mathbf{K} \rightarrow \mathscr{F} \mathcal{C}$, where $\mathbf{K}$ is a certain finite ordered simplicial complex, considered as a category, and where $\mathscr{F} \mathcal{C}$ is the category of smooth compact manifolds and smooth finite covering maps. $\mathbf{K}$ is obtained as the barycentric subdivision of some triangulation of the finite complex $X_{S} / \Gamma$ where $X_{S}$ is the Bruhat-Tits factor of $X$. That is, fix a triangulation of $X_{S} / \Gamma$. Then the vertices of $\mathbf{K}$ correspond bijectively with the simplices of $X_{S} / \Gamma$. The functor $F$ is obtained in the following way; let $\tau \subset \sigma$ be simplices of the fixed triangulation of $X_{S} / \Gamma$. Put $F(\sigma)=X_{\infty} / \Gamma_{\sigma}$ where $X_{\infty}$ is the "classical" factor of $X$, and $\Gamma_{\sigma}$ is the stabiliser subgroup of $\sigma$ in $\Gamma$. The obvious map $F(\sigma) \rightarrow F(\tau)$ is a finite covering map, and is clearly smooth. This description of $X / \Gamma$ is on pp. 230-231 of [2].

To complete the proof, it is enough to show that if $\mathbf{K}$ is any finite ordered simplicial complex, considered as a category in the same way we considered the $n$-cube $2^{n}$ as a category, then for any functor $F: \mathbf{K} \rightarrow \mathscr{F} \mathcal{C},\langle F\rangle$ is a stratified set. If * denotes the trivial diagram of points over $\mathbf{K}$, then $\langle *\rangle \cong|\mathbf{K}|$, the geometrical realisation of $\mathbf{K}$ as a simplicial complex. The obvious natural transformation $F \rightarrow *$ induces a surjection $p:\langle F\rangle \rightarrow|\mathbf{K}| .|\mathbf{K}|$ is naturally stratified with open simplices as strata. The stratification on $\langle F\rangle$ is the obvious one in which the strata of $\langle F\rangle$ are the preimages of the strata of $|\mathbf{K}|$. To check that this stratification is a genuine one in the sense of $[\mathbf{1 0}, \mathbf{1 5}]$, it suffices to check that the product of an attaching map with a smooth locally trivial fibre bundle is still an attaching map, which is obvious. Q.E.D.

Finally, our results can be used to complete and correct the proof of Yang's Triangulation Theorem [19].

If $M \times G \rightarrow M$ is a smooth transformation group and $M, G$ are compact, it is a folk theorem that $M / G$ is a stratified set, though the literature still seems to lack an 
explicit proof of this fact. The usual incantation is "using the Slice Theorem, we see that $M / G$ is a stratified set". Allowing this, Theorem 2.1 above completes the proof of Yang's Theorem that $M / G$ is triangulable. In the case where $G$ is finite, Yang's Theorem has been reproved ab initio by Illman [6].

\section{REFERENCES}

1. A. Borel and J. P. Serre, Corners and arithmetic groups, Comment. Math. Helv. 48 (1973), 436-491.

2. C__ Cohomologie d'immeubles et de groupes S-arithmétiques, Topology 15 (1976), 211-232.

3. J. Cerf, Topologie de certains espaces de plongements, Bull. Soc. Math. France 89 (1961), 227-380.

4. A. Douady and L. Herault, Appendix to [1].

5. H. Hironaka, Triangulation of algebraic sets, Proc. Amer. Math. Soc. Inst. Algebra Geom. Arcata (1974).

6. S. Illman, Smooth equivariant triangulations of $G$-manifolds for $G$ a finite group, Math. Ann. 233 (1978), 199-220.

7. F. E. A. Johnson, Triangulation of stratified sets, Thesis, University of Liverpool, 1972.

8. _ A triangulation criterion, Mathematika 25 (1978), 110-114.

9. __ On the triangulation of smooth fibre bundles, Fund. Math. (to appear).

10. On the presentation of stratified sets and singular varieties, Mathematika (to appear).

11. B. O. Koopman and A. B. Brown, On the covering of analytic loci by complexes, Trans. Amer. Math. Soc. 34 (1932), 231-251.

12. S. Lefschetz and J. H. C. Whitehead, On analytical complexes, Trans. Amer. Math. Soc. 35 (1933), 510-517.

13. S. Lojasiewicz, Triangulation of semi-analytic sets, Ann. Sculoa Norm. Sup. Pisa Cl. Sci. 18 (1964), 449-473.

14. B. Teissier, Théorèmes de finitude en géométrie analytique (d'après Heisuke Hironaka), Sém. Bourbaki 1973/74, no. 451, Lecture Notes in Math., vol. 431, Springer-Verlag, Berlin and New York, 1975.

15. R. Thom, Ensembles et morphismes stratifiés, Bull. Amer. Math. Soc. 75 (1969), 240-284.

16. B. L. Van der Waerden, Topolgische Begründung des Kalküls der abzählen Geometrie, Math. Ann. 102 (1929), 360.

17. J. H. C. Whitehead, On $C^{1}$ complexes, Ann. of Math. (2) 41 (1940), 809-824.

18. H. Whitney, Tangents to an analytic variety, Ann. of Math. (2) 81 (1965), 496-549.

19. C. T. Yang, The triangulability of the orbit space of a differentiable transformation group, Bull. Amer. Math. Soc. 69 (1963), 405-408.

Department of Mathematics, University College London, London WC1E 6BT, England 